\title{
Newtonian noise limit in atom interferometers for gravitational wave detection
}

\author{
Flavio Vetrano $^{1,2, a}$, Andrea Viceré ${ }^{1,2, b}$ \\ ${ }^{1}$ Dipartimento di Scienze di Base e Fondamenti-DiSBeF, Università degli Studi di Urbino "Carlo Bo", 61029 Urbino, Italy \\ ${ }^{2}$ INFN, Sezione di Firenze, INFN, 50019 Sesto Fiorentino, Italy
}

Received: 10 June 2013 / Revised: 17 September 2013 / Published online: 9 October 2013

(C) The Author(s) 2013. This article is published with open access at Springerlink.com

\begin{abstract}
In this work we study the influence of the Newtonian noise on atom interferometers applied to the detection of gravitational waves, and we compute the resulting limits to the sensitivity in two different configurations: a single atom interferometer, or a pair of atom interferometers operated in a differential configuration. We find that for the instrumental configurations considered, and operating in the frequency range [0.1-10] Hz, the limits would be comparable to those affecting large scale optical interferometers.
\end{abstract}

\section{Introduction}

The direct detection of Gravitational Waves is one of the most exciting challenges of current scientific research. The first generation of ground-based optical interferometric detectors, including Virgo [1] and GEO600 [2] in Europe, and the LIGO [3] interferometers in USA, achieved design sensitivity and carried out several science runs, which set interesting upper limits on several classes of astrophysical sources [4-7]. The construction of a "second generation" of optical interferometers, Advanced LIGO [8] and Virgo [9], and the new Japanese detector KAGRA [10], is well underway; thanks to the implementation of several technical upgrades, the advanced detectors are expected to come on line with a sensitivity about ten times better than first generation instruments. In the meanwhile, the conceptual design of third generation detectors, like the Einstein Telescope $[11,12]$, has started.

For all these optical ground based detectors the sensitivity in the low frequency band, below $10 \mathrm{~Hz}$, is ultimately limited by the so called "gravity gradient", or Newtonian

\footnotetext{
a e-mail: flavio.vetrano@uniurb.it
}

b e-mail: andrea.vicere@uniurb.it
Noise (NN) [13, 14], whose source is the direct coupling of the test masses with any mass-density change in the environment, especially of seismic or atmospheric origin.

Atom interferometers (see [15] for a review) have been proposed recently as GW detectors [16-21], on the basis of previous general ideas [22]. These instruments promise to be less sensitive to some of the noise sources affecting optical instruments: for instance, being the atoms in free fall, no direct seismic noise should be present. The effect of gravitational waves is a change in the phase accumulated by atoms' wave functions, which can be detected by observing the interference of two atom beams.

However, also the non-radiative gravitational fields of terrestrial origin affect the phase, in a different way as we will show: the question arises then, if the "low frequency wall" due to $\mathrm{NN}$ is relevant also for these new proposed detectors. In this paper we consider only the $\mathrm{NN}$ of seismic origin and we carry out a detailed calculation of its contribution to the sensitivity curve of an atom interferometer both in the "single detector" configuration and in the "coupled differential" configuration.

It is worth underlining that this study is motivated by the different way in which gravitational fluctuations couple to atom interferometers and to optical interferometers, related to the fact that in the first case the test masses are atoms freely traveling across the instrument. We anticipate our conclusions: the atom interferometers are subject to NN in a degree similar to optical interferometers, and therefore will require appropriate technical solutions to overcome this noise limit in the frequency band below $10 \mathrm{~Hz}$.

The paper is organized as follows: in Sect. 2 we consider a definite atom interferometer and we compute its response to a fluctuating gravity field; in Sect. 3 we apply the formulas to the case of a single detector, deriving the limits on sensitivity; finally in Sect. 4 we consider two atom interferometers operated in differential configurations. 


\section{Newtonian noise of seismic origin in atom interferometers}

In optical interferometric $\mathrm{GW}$ detectors the test masses are suspended mirrors: a pendular suspension is indeed the best approximation on Earth for a freely falling test mass. In atom interferometers instead the role of test masses is played by atoms in free fall, hence our intent is to determine the influence of the Newtonian coupling to an external, timevarying mass distribution, on freely falling masses.

Some general considerations are possible: if the effect originates from seismic noise, it is driven by an external masses displacement field, whose linear power spectral density will generally have the form $\tilde{W}(\omega) \sim \omega^{-2}$, mediated by a transfer function from the seism to the test masses motion behaving also as $\omega^{-2}[14,23,24]$, where $\omega$ is the angular frequency. Therefore the effect on test masses is expected to be of the form $\theta(\omega) \Gamma \omega^{-4}$, hence more relevant at low frequencies, where $\theta(\omega)$ is a kind of reduced transfer function, depending on the detection device, and $\Gamma$ is a scale factor depending on the model of seismic waves (it is recognized that the role of main source is played by Rayleigh surface waves, especially the fundamental mode and few overtones [23, 24]).

To derive the actual expression of $\theta(\omega)$ for $\mathrm{NN}$ in an atom interferometer, we use the ABCD formalism for matter waves, described elsewhere in detail [20, 25].

Assume that the Hamiltonian of the motion for the atoms is at most quadratic in momentum and position operators

$$
\begin{aligned}
H= & \sum_{n, r=1}^{3}\left[\frac{1}{2 M} p_{n} \beta_{n r}(t) p_{r}+\frac{1}{2} p_{n} \alpha_{n r}(t) q_{r}-\frac{1}{2} q_{n} \delta_{n r}(t) p_{r}\right. \\
& \left.-\frac{M}{2} q_{n} \gamma_{n r}(t) q_{r}+f_{n}(t) p_{n}-M g_{n}(t) q_{n}\right]
\end{aligned}
$$

where $p_{n(r)}$ and $q_{n(r)}$ are vectors of momentum and position, respectively, whereas $\alpha, \beta, \gamma, \delta$ are suitable square matrices (note that $\delta=-\alpha^{T}$, with $T$ indicates the transposed matrix), and $M$ is the atom rest mass.

The last term in the Hamiltonian represents the response to the local, fluctuating gravitational field $\mathbf{g}(t)$ : in the following, we will consider only the component along the direction of motion of the atoms, as in the paraxial approximation all transverse effects are neglected. The $\gamma$ term allows to model the response to gravitational waves: in the Fermi gauge, and considering Fourier components, one can show that $\hat{\gamma}=\frac{\omega^{2}}{2} \hat{h}(\omega)$, where $\hat{h}(\omega)$ is the gravitational wave strain tensor (see for instance [20]).

Consider an atoms' beam (a Gaussian packet under paraxial approximation [20, 25-28]) which is divided and recombined through a sequence of $R$ light-field beam splitters, supplied by the same laser: from the first beam splitter to the last one (the output port) we may identify two paths, conventionally labeled $s$ and $i$. By exploiting the ttt theorem [25] for the atoms/beam splitter interactions, and the mid-point property of Gaussian beams [29], the phase difference at the output port of the interferometer can be written as:

$$
\begin{aligned}
\Delta \phi= & \sum_{j=1}^{R}\left[\left(k_{s j}-k_{i j}\right) \frac{q_{s j}+q_{i j}}{2}-\left(\omega_{s j}-\omega_{i j}\right) t_{j}\right. \\
& \left.+\left(\theta_{s j}+\theta_{i j}\right)\right]
\end{aligned}
$$

where $k_{s(i) j}$ is the momentum transferred to the atoms by the $j$ th beam splitter along the $s(i)$ arm, $\omega_{s(i) j}$ is the angular frequency of the laser beam and $\theta_{s(i) j}$ is the phase of the laser beam at the $j$ th interaction, $q_{s(i) j}$ is the distance of $j$ th interaction point from the laser source; equal masses are assumed for the atoms along the $s$ and $i$ paths. The expression in Eq. (2) is manifestly gauge-invariant [20, 25], and the evolution of the wave packets can be obtained, by means of the Ehrenfest theorem, from Hamilton's equations for the vector $\chi(t)[20,25,28]$

$\frac{d \chi}{d t}=\left(\begin{array}{c}\frac{d H}{d p} \\ -\frac{1}{M} \frac{d H}{d q}\end{array}\right)=\Gamma(t) \cdot \chi(t)+\Phi(t)$,

where

$\chi \equiv\left(\begin{array}{c}q \\ \frac{p}{M}\end{array}\right) ; \quad \Phi(t) \equiv\left(\begin{array}{c}f(t) \\ g(t)\end{array}\right) ;$

$\Gamma(t) \equiv\left(\begin{array}{ll}\alpha(t) & \beta(t) \\ \gamma(t) & \delta(t)\end{array}\right)$

in the form

$\chi(t)=\left(\begin{array}{ll}A\left(t, t_{0}\right) & B\left(t, t_{0}\right) \\ C\left(t, t_{0}\right) & D\left(t, t_{0}\right)\end{array}\right) \cdot\left[\chi\left(t_{0}\right)+\left(\begin{array}{c}\xi\left(t, t_{0}\right) \\ \psi\left(t, t_{0}\right)\end{array}\right)\right]$,

where

$\left(\begin{array}{ll}A\left(t, t_{0}\right) & B\left(t, t_{0}\right) \\ C\left(t, t_{0}\right) & D\left(t, t_{0}\right)\end{array}\right)=\tau \exp \left[\int_{t_{0}}^{t} \Gamma\left(t^{\prime}\right) d t^{\prime}\right]$,

$\left(\begin{array}{c}\xi\left(t, t_{0}\right) \\ \psi\left(t, t_{0}\right)\end{array}\right)=\int_{t_{0}}^{t}\left(\begin{array}{ll}A\left(t_{0}, t^{\prime}\right) & B\left(t_{0}, t^{\prime}\right) \\ C\left(t_{0}, t^{\prime}\right) & D\left(t_{0}, t^{\prime}\right)\end{array}\right) \cdot \Phi\left(t^{\prime}\right) d t^{\prime} ;$

here $\tau$ represents the time-ordering operator, and an appropriate perturbative expansion can be used to evaluate the time-ordered exponential in Eq. (6) [20, 25, 28].

As a simple reference configuration let us consider a "Ramsey-Bordé" atom interferometer, with a MachZehnder geometry, as outlined in Fig. 1 [15, 20, 25]. In the following, we will also assume that the instrument is crossed by a plane GW with "+" polarization and amplitude $h$, propagating along the $x_{3}=z$ axis, perpendicular to 


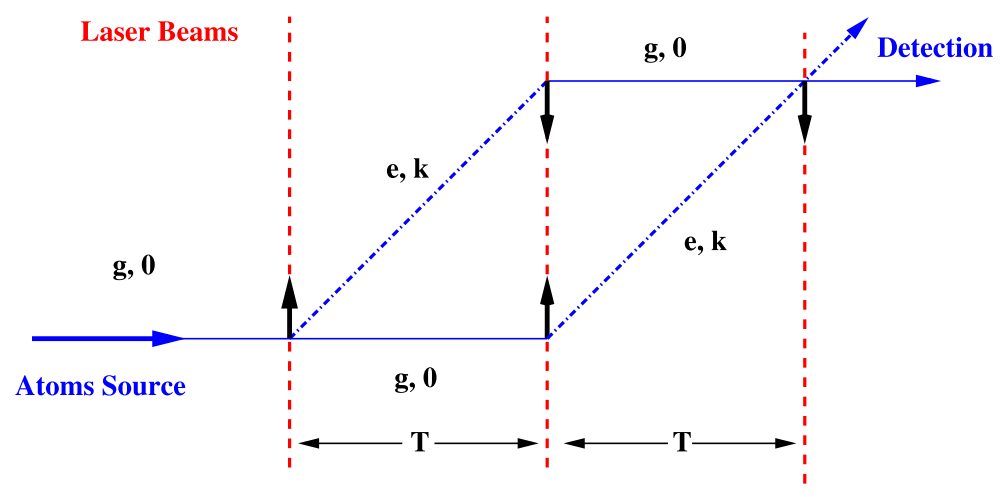

Fig. 1 A simple "Ramsey-Bordé atom interferometer with MachZehnder geometry. Continuous horizontal lines, and the slanted dotdashed lines, represent atom beams. Vertical dashed lines represent the

the plane of the interferometer; we adopt in the following a description in Fermi coordinates, which represents the best approximation to the Laboratory Cartesian system [30].

Assuming the same "stable" frequency for the laser beams and neglecting the steady proper laser phases, the phase shift formula in Eq. (2) becomes

$\Delta \phi=\sum_{j=1}^{4}\left(k_{s j}-k_{i j}\right) \frac{q_{s j}+q_{i j}}{2}$.

Let us assume that atoms are subjected only to a fluctuating gravitational field $g(t)$. Considering Eqs. (1), (3), (4) and (7) we have

$\alpha=\delta=\gamma=0 ; \quad \beta=1 ; \quad f(t)=0 ; \quad g(t) \neq 0 ;$

$A=1 ; \quad B=t-t_{0} ; \quad C=0 ; \quad D=1 ;$

and we obtain

$$
\left(\begin{array}{c}
\xi\left(t, t_{0}\right) \\
\psi\left(t, t_{0}\right)
\end{array}\right)=\int_{t_{0}}^{t}\left(\begin{array}{c}
t_{0}-t^{\prime} \\
t^{\prime}
\end{array}\right) g\left(t^{\prime}\right) d t^{\prime}
$$

We are interested in the low frequency range, where the Newtonian noise is expected to be the limiting factor on account of its $\omega^{-4}$ shape. We will therefore assume that the single atom interferometer has a linear dimension smaller than the wavelength of seismic surface waves, which we will assume to set also the coherence length. Introducing the Fourier transform $\hat{g}(\omega)$ of the fluctuating field we can also write

$$
\xi\left(t, t_{0}\right)=\int \frac{d \omega}{2 \pi} \hat{g}(\omega)\left[-\frac{\left(t-t_{0}\right)}{i \omega} e^{i \omega t}-\frac{1}{\omega^{2}}\left(e^{i \omega t}-e^{i \omega t_{0}}\right)\right],
$$$$
\psi\left(t, t_{0}\right)=\int \frac{d \omega}{2 \pi} \hat{g}(\omega)\left[\frac{e^{i \omega t_{0}}}{i \omega}\left(e^{i \omega\left(t-t_{0}\right)}-1\right)\right]
$$

and we assume, in the long wavelength approximation, that $\hat{g}(\omega)$ is the same at any point of the interferometer. There- laser beams; the bold continuous arrows represent relevant momentum transferred to the atoms; $g$ and $e$ mark the ground and excited internal states of the atoms; $\mathbf{k}$ is the transverse momentum in $\hbar$ units

fore the solution of the Hamilton equations Eq. (5) can be written as

$$
\begin{aligned}
& \left(\begin{array}{c}
q(t) \\
\frac{p(t)}{M}
\end{array}\right) \\
& =\left(\begin{array}{cc}
1 & t-t_{0} \\
0 & 1
\end{array}\right) \cdot\left[\left(\begin{array}{c}
q\left(t_{0}\right) \\
\frac{p\left(t_{0}\right)}{M}
\end{array}\right)\right. \\
& \left.\quad+\int \frac{d \omega}{2 \pi} \hat{g}(\omega)\left(\begin{array}{c}
-\frac{\left(t-t_{0}\right)}{i \omega} e^{i \omega t}-\frac{1}{\omega^{2}}\left(e^{i \omega t}-e^{i \omega t_{0}}\right) \\
\frac{e^{i \omega t}}{i \omega}\left(e^{i \omega\left(t-t_{0}\right)}-1\right)
\end{array}\right)\right]
\end{aligned}
$$

this expression allows to compute the values of the coordinates and momenta of the atoms at the interaction points with the laser: by iterating the relation in Eq. (5) to the four interaction points of the interferometer in Fig. 1, setting $t_{3}=t_{2}$ and defining $T=t_{4}-t_{3}=t_{2}-t_{1}$, we finally obtain the phase shift at the output port of the interferometer:

$\Delta \hat{\phi}(\omega)=k T^{2} e^{i \omega T}\left[\frac{\sin (\omega T / 2)}{(\omega T / 2)}\right]^{2} \hat{g}(\omega)$

this is the fundamental formula to estimate the effect of the fluctuating field $\hat{g}$. We recall that $k$ is the unperturbed wave vector of the laser beam, corresponding to the impulse (in units of the reduced Planck constant $\hbar$ ) transferred to the atom at each interaction point. Note also that in the limit $\omega \rightarrow 0$ the expression in Eq. (13) corresponds to the well known static result $[29,31]$.

\section{Newtonian-Noise limit on sensitivity: the single detector case}

In the weak field approximation, to first order in the amplitude $h$ of an impinging gravitational wave, the phase shift at the output of the interferometer in Fig. 1 has been already 
obtained in a fully covariant way [20]. Indicating with $q_{1}$ the unperturbed distance of the first interaction point from the laser, and with $p_{1}$ the unperturbed momentum of the atoms, just before the first interaction with the laser beam, we recall that the Fourier transform of the phase shift, as a function of the Fourier transformed amplitude $\hat{h}$ of the GW, can be written as

$$
\begin{aligned}
\Delta \hat{\phi}(\omega)= & \omega \hat{h}(\omega) \frac{T^{2} k}{M}\left(p_{1}+\frac{k \hbar}{2}\right) \\
& \times\left[\frac{e^{i \omega T}-e^{2 i \omega T}}{\omega T}+i e^{i \omega T}\left(\frac{\sin (\omega T / 2)}{\omega T / 2}\right)^{2}\right] \\
& +\frac{\omega^{2} \hat{h}(\omega)}{2} T^{2} k q_{1}\left(\frac{\sin (\omega T / 2)}{\omega T / 2}\right)^{2} e^{i \omega T}
\end{aligned}
$$

in which the proper laser phases have been neglected.

Comparing with the expression of the response to a fluctuating local gravity field Eq. (13), we note that the second term of Eq. (14) corresponds to it, with the substitution $\tilde{g} \rightarrow \frac{q_{1}}{2} \omega^{2} \tilde{h}$ : however, the overall response to GWs includes also a dynamic term depending on the atom momentum $p_{1}$ and on the momentum $k$ transferred to the atoms: hence the effects of the local gravitational field and of the gravitational waves are in principle distinguishable.

For a single interferometer with the laser source close to the device, actually the last term can be neglected and the more relevant one is the term proportional to $p_{1}$, since we can also generally neglect the recoil term $\frac{k \hbar}{2 M}$. This expression can be directly translated into a relation between linear power spectral densities (LPSD), that we denote by a tilde, defined in terms of the two-point correlation functions as

$\left\langle\hat{g}(\omega) \hat{g}\left(\omega^{\prime}\right)\right\rangle=2 \pi \delta\left(\omega-\omega^{\prime}\right) \tilde{g}^{2}(\omega)$

in which the angular brackets represent the statistical average. From Eq. (13) and Eq. (14) we obtain

$$
\begin{aligned}
\Delta \tilde{\phi}(\omega)= & \tilde{h}(\omega) k L|\sin (\omega T / 2)| \\
& \times \sqrt{1-\frac{2 \sin (\omega T)}{\omega T}+\left[\frac{\sin (\omega T / 2)}{(\omega T / 2)}\right]^{2},} \\
\Delta \tilde{\phi}(\omega)= & k T^{2}\left[\frac{\sin (\omega T / 2)}{(\omega T / 2)}\right]^{2} \tilde{g}(\omega),
\end{aligned}
$$

where the distance $L=2 T p_{1} / M$ travelled by the atoms in the interferometer of Fig. 1 has been introduced; combining the two equations, we deduce the expression

$$
\tilde{h}_{\mathrm{NN}}(\omega)=\frac{4}{\omega^{2}} \frac{|\sin (\omega T / 2)|}{\sqrt{1-\frac{2 \sin (\omega T)}{\omega T}+\left[\frac{\sin (\omega T / 2)}{(\omega T / 2)}\right]^{2}}} \frac{\tilde{g}(\omega)}{L}
$$

for the equivalent strain $\tilde{h}_{\mathrm{NN}}$ induced by the fluctuating field $\tilde{g}(\omega)$.
It is useful to discuss here the scale of the $\tilde{g}(\omega)$ LPSD, referring to typical values measured at the site of the Virgo interferometers; we recall indeed that we are considering the effect of an external fluctuating gravity field on freely falling test masses, which is the same situation experienced by the test masses of optical interferometers [14, 23, 24]; even though the detailed shape of the $\mathrm{NN}$ affecting a instrument like Virgo depends on the model for the seismic sources and the superficial Earth layers, similar results are obtained in different cases, which can be summarized as follows

$\tilde{h}_{\mathrm{NN}}(\omega)=\frac{\sqrt{4} \tilde{X}(\omega)}{L_{V}} \simeq \frac{1.2 \times 10^{-9}}{\omega^{2}} \tilde{x}_{\mathrm{seism}}(\omega) \times \frac{H z^{2}}{m}$,

where $L_{V}=3000 \mathrm{~m}$ is the length of Virgo arms, $\tilde{X}(\omega)$ is the displacement LPSD for a single suspended mirror, and $\tilde{x}_{\text {seism }}(\omega)$ is the measured LPSD of the ground seism [32]; the factor $\sqrt{4}$ takes into account that in Virgo the noise due to the four end-station mirrors adds in quadrature.

Considering the relation between the mirror motion and its acceleration, due to the fluctuating gravitational field, $\tilde{g}(\omega)=\omega^{2} \tilde{X}(\omega)$, we obtain

$$
\begin{aligned}
\frac{\tilde{g}(\omega)}{L} & =\frac{\omega^{2} L_{V}}{2 L} \frac{\sqrt{4} \tilde{X}(\omega)}{L_{V}} \\
& \simeq 6 \times 10^{-10} \frac{L_{V}}{L} \tilde{x}_{\text {seism }}(\omega) \times \frac{\mathrm{Hz}^{2}}{\mathrm{~m}}
\end{aligned}
$$

we further assume that the seismic noise measured at the Virgo site is well approximated by [33]

$\tilde{x}_{\text {seism }}(\omega) \simeq \frac{10^{-7}}{[\omega /(2 \pi \mathrm{Hz})]^{2}} \mathrm{mHz}^{-1 / 2} ;$

Following [28], let us assume very ambitious parameters for the single Ramsey-Bordé atom interferometer: a length $L \sim 200 \mathrm{~m}$, which could result in interesting sensitivities to gravitational waves, and a time of flight $T=0.4 \mathrm{~s}$, in order to have not too small a bandwidth; obviously the choice implies atom speeds of the order of $250 \mathrm{~m} / \mathrm{s}$, and we underline that such choices are probably beyond the limits of current technologies. Anyway, we obtain

$\frac{\tilde{g}(\omega)}{L} \sim \frac{10^{-16}}{[\omega /(2 \pi \mathrm{Hz})]^{2}} \mathrm{~Hz}^{2}$.

as an estimate of the scale of the fluctuating gravitational field seen by the atom interferometer.

To appreciate the result, we show in Fig. 2 an example of the Newtonian noise of Eq. (17) assuming the expression in Eq. (21) for the LPSD of the fluctuating gravitational 


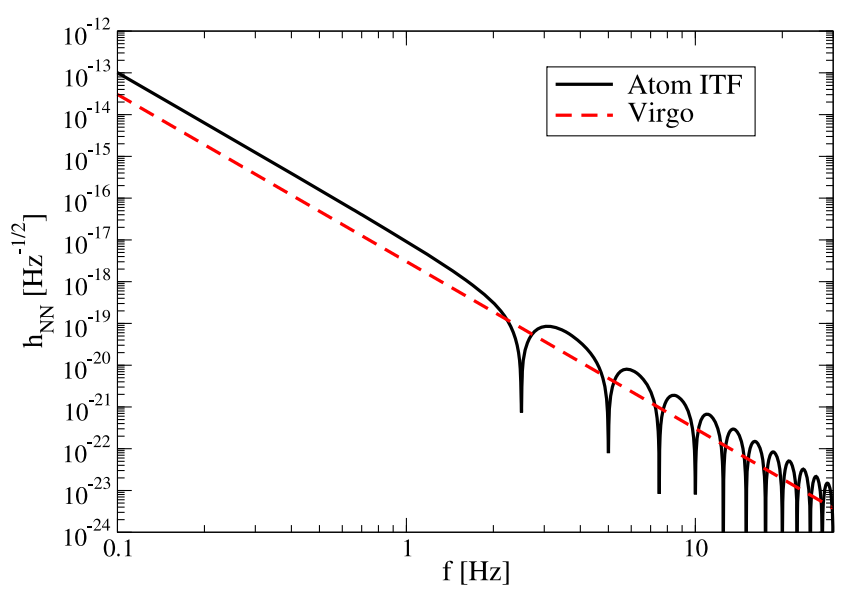

Fig. 2 The solid curve represents the effect of the gravity gradient noise on a single atom interferometer, with the expected $\omega^{-4}$ behavior, and zeroes corresponding to frequencies at which the instrument is insensitive both to the gravity gradient fluctuations and to gravitational waves. For comparison, the dashed curve represents the model Newtonian noise effect on the Virgo interferometer

field; in the same figure we plot, for comparison, the corresponding Newtonian noise for the Virgo detector. ${ }^{1}$

The zeroes represent frequencies at which the atom interferometer is insensitive both to the gravity gradient noise and to GW; note that the one shown is not a complete noise budget, to which other noises would contribute, particularly the atom shot noise which would exhibit peaks at those frequencies, not differently from an optical interferometer in a Michelson configuration and without Fabry-Perot cavities.

Apart this specific feature, the comparison with a large optical interferometer shows a similar behavior as a function of the frequency, with a different noise scale dictated by the different linear dimensions of the instruments. We underline that for this type of atom interferometer, it could be unrealistic to increase the linear size $L$ even further: to this end, a differential configuration appears more promising.

\section{Two detectors operated in a differential configuration}

Let us now consider the second term in Eq. (14), proportional to the position $q_{1}$. This term, already introduced in a different context [34], is a sort of "clock" term which takes into account the influence of the GW on the laser beam, along its path from the source to a well defined physical point. Its role was discussed in recent papers [35-37] and the most relevant new property is the introduction of $q_{1}$ (path of laser beam) in place of $L$ (path of atom beam); so, in order

\footnotetext{
${ }^{1}$ It should be underlined that in this low frequency band, below $10 \mathrm{~Hz}$, the actual noise of Virgo is dominated by other noise sources, most notably by the direct seismic noise and by the thermal noise, not to mention other technical noises.
}

to improve the sensitivity, enlarging $q_{1}$ seems in principle easier than enlarging $L$.

This solution requires measuring the distance from the laser, and carries additional requirements on the coherence and stability of the laser beam, while maintaining it at a sufficient power density: it is therefore premature to draw too optimistic conclusions about the practicality of the configuration. However, the idea of adopting a two-interferometers differential configuration [21] appears very appealing in order to render the system independent from the laser position, and may furthermore yield a good common-modes rejection.

Under the hypothesis of a common laser source for two identical Mach-Zehnder atom interferometers in differential configuration, for which the relative distance $D$ satisfies the condition $\omega D / c \ll 1$ (with $c$ the speed of light in vacuum), from Eq. (14) the overall difference between the two partial phase differences at the output ports can be formally obtained as

$\Delta \hat{\phi}(\omega)=2 k D \sin ^{2}(\omega T / 2) e^{i \omega T} \hat{h}(\omega)$

where $D \equiv q_{1}^{\mathrm{II}}-q_{1}^{\mathrm{I}}$ as anticipated. Considering also Eq. (13) we obtain for the differential configuration

$\hat{h}_{\mathrm{NN}}(\omega)=\frac{2}{\omega^{2} D}\left[\hat{g}_{2}(\omega)-\hat{g}_{1}(\omega)\right]$,

where the difference in the right hand side requires some discussion. In a given frequency band, if the two fluctuating gravity fields $\hat{g}_{1,2}$ act upon sufficiently distant atom interferometers, they will be uncorrelated, and we will obtain for the LPSD simply a sum in quadrature

$\tilde{h}_{\mathrm{NN}}(\omega)=\frac{2}{\omega^{2} D} \sqrt{\tilde{g}_{1}^{2}(\omega)+\tilde{g}_{2}^{2}(\omega)}$

displaying no conceptual difference with respect to the limits obtained for optical interferometers with long arms [32]. Considering instead a low-frequency, long-wavelength approximation, it may be appealing the situation in which, even with two separated interferometers, the residual correlation leads to a partial noise cancellation in Eq. (23).

We recall that the signals $\hat{g}_{1,2}(\omega)$ are assumed to be stochastic acceleration fields in positions 1 and 2, projected along the direction specified by the segment $\mathbf{D}$ as in Fig. 3 .

We further assume to model the stochastic noise in the simplest possible way, namely as due to uncorrelated fluctuations in the density of the material surrounding the detector [14]. In other words a density fluctuation $\Delta M(t)$ will contribute to the acceleration field in points 1 and 2 as

$$
\begin{aligned}
& \mathbf{g}_{2}(t)=\frac{G \Delta M(t)}{r_{2}^{2}} \hat{r}_{2}=\frac{G \Delta M(t)}{r_{2}^{3}} \mathbf{r}_{2}, \\
& \mathbf{g}_{1}(t)=\frac{G \Delta M(t)}{r_{1}^{2}} \hat{r}_{1}=\frac{G \Delta M(t)}{\left|r_{2}-D\right|^{3}}\left(\mathbf{r}_{2}-\mathbf{D}\right) .
\end{aligned}
$$




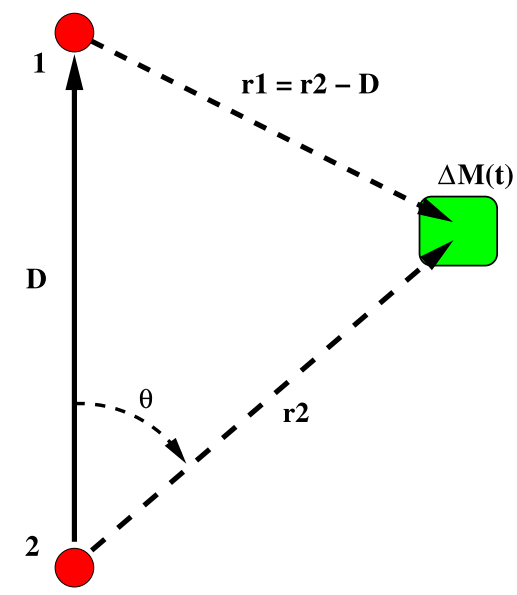

Fig. 3 Geometry of the detector: atom interferometers are located at positions 1 and 2 , and a fluctuating mass element is assumed at a location $\mathbf{r}_{2}$ in a frame having position 2 as the origin, and a $\hat{z}$ axis parallel to $\mathbf{D}$

Considering only the component acting along the direction separating the two points 1 and 2, we obtain

$g_{2}(t)=\frac{G \Delta M(t)}{r^{2}} \cos (\theta)$,

$g_{1}(t)=\frac{G \Delta M(t)}{\left[r^{2}+D^{2}-2 r D \cos (\theta)\right]^{3 / 2}}[r \cos (\theta)-D]$

as the contribution to the fluctuation of the acceleration field due to a single mass element. To obtain the total fluctuation, we need now to sum over the space.

We first assume for simplicity that the space around the two stations with atom interferometers can be considered homogeneous: this could be the case for instance if the instrumentation is placed in a deep mine, at a depth much larger than $D$. We are therefore interested in the quantity

$$
\begin{aligned}
\hat{h}_{\mathrm{NN}}(\omega, \mathbf{r})= & \frac{2}{\omega^{2} D}\left[\hat{g}_{2}(\omega)-\hat{g}_{1}(\omega)\right] \\
= & \frac{2 G \Delta M(\omega, \mathbf{r})}{\omega^{2} D}\left\{\frac{\cos (\theta)}{r^{2}}\right. \\
& \left.-\frac{r \cos (\theta)-D}{\left[r^{2}+D^{2}-2 r D \cos (\theta)\right]^{3 / 2}}\right\}
\end{aligned}
$$

which should be summed over the volume. It is convenient to evaluate the spectral density

$$
\begin{aligned}
\left\langle h_{\mathrm{NN}}(\omega) h_{\mathrm{NN}}\left(\omega^{\prime}\right)\right\rangle & \equiv 2 \pi \delta\left(\omega-\omega^{\prime}\right) \tilde{h}_{\mathrm{NN}}^{2}(\omega) \\
& =\sum_{\mathbf{r}, \mathbf{r}^{\prime}}\left\langle\Delta h_{\mathrm{NN}}(\omega, \mathbf{r}) \Delta h_{\mathrm{NN}}\left(\omega^{\prime}, \mathbf{r}^{\prime}\right)\right\rangle
\end{aligned}
$$

where, following again Saulson [14], we assume the sum to be extended over volume elements of linear size $\lambda / 2$, with $\Delta M$ fluctuating coherently inside these regions, and totally uncorrelated otherwise:

$$
\left\langle\Delta M(\omega, \mathbf{r}) \Delta M\left(\omega^{\prime}, \mathbf{r}^{\prime}\right)\right\rangle=2 \pi \delta\left(\omega-\omega^{\prime}\right) \Delta \tilde{M}^{2}(\omega, \mathbf{r}) \delta_{\mathbf{r}, \mathbf{r}^{\prime}}
$$

We obtain therefore

$$
\begin{aligned}
\tilde{h}_{\mathrm{NN}}^{2}(\omega)= & \frac{4 G^{2}}{\omega^{4} D^{2}} \sum_{\mathbf{r}} \Delta \tilde{M}^{2}(\omega, \mathbf{r})\left\{\frac{\cos (\theta)}{r^{2}}\right. \\
& \left.-\frac{r \cos (\theta)-D}{\left[r^{2}+D^{2}-2 r D \cos (\theta)\right]^{3 / 2}}\right\}^{2} .
\end{aligned}
$$

If we additionally assume that the mass fluctuations do not depend on $\mathbf{r}$, we can further simplify, obtaining

$$
\begin{aligned}
\tilde{h}_{\mathrm{NN}}^{2}(\omega)= & \frac{4 G^{2} \Delta \tilde{M}^{2}(\omega)}{\omega^{4} D^{2}} \sum_{\mathbf{r}}\left\{\frac{\cos (\theta)}{r^{2}}\right. \\
& \left.-\frac{r \cos (\theta)-D}{\left[r^{2}+D^{2}-2 r D \cos (\theta)\right]^{3 / 2}}\right\}^{2} \\
= & \frac{4 G^{2} \Delta \tilde{M}^{2}(\omega)}{\omega^{4} D^{2}}\left(\frac{2}{\lambda}\right)^{3} \int\left\{\frac{\cos (\theta)}{r^{2}}\right. \\
& \left.-\frac{r \cos (\theta)-D}{\left[r^{2}+D^{2}-2 r D \cos (\theta)\right]^{3 / 2}}\right\}^{2} r^{2} d r d \cos \theta d \phi,
\end{aligned}
$$

where we have approximated the sum with an integral, normalizing by the volume element of the coherent region $(\lambda / 2)^{3}$. If we were to retain only the first term, we would obtain the same result as in [14], corrected for a factor 2 which is wrong in the original paper. The integration over the angular functions is directly carried out, resulting in a lengthy expression:

$$
\begin{aligned}
& \tilde{h}_{\mathrm{NN}}^{2}(\omega)=\frac{64 \pi G^{2} \Delta \tilde{M}^{2}(\omega)}{\omega^{4} D^{2} \lambda^{3}} \cdot H(D, \lambda), \\
& H=\int \frac{r\left\{4\left[8(D-r)^{2}(D+r)^{2}+3 D r\left(3 D^{2}-r^{2}\right)\right]-3\left(D^{2}-r^{2}\right)^{2} \ln \frac{(D-r)^{2}}{(D+r)^{2}}\right\}}{24 D^{3}(D-r)^{2}(D+r)^{2}} d r+\int \frac{2\left(D^{3}+2 r^{3}\right)(D-r)}{3 D^{3} r^{2}|D-r|} d r,
\end{aligned}
$$


which, as expected, displays double poles in $r=0$ and in $r=D$.

Both divergences are artefacts, which should be regulated introducing cutoffs $r \geq \frac{\lambda}{4}$ and at $|r-D| \geq \frac{\lambda}{4}$. However, it is now necessary to distinguish two cases

Short wavelength If the distance $D \gg \lambda$, then the integral over $r$ gives

$H(D, \lambda)=\frac{14}{3 \lambda}+O\left(\frac{\lambda}{D^{2}} \ln \frac{\lambda}{D}\right)$

and we obtain

$\tilde{h}_{\mathrm{NN}(s w)}^{2}(\omega) \simeq \frac{896 \pi G^{2} \Delta \tilde{M}^{2}(\omega)}{3 \omega^{4} D^{2} \lambda^{4}}$.

Long wavelength In the long wavelength approximation the integral in Eq. (33) can be carried out assuming $r \geq \frac{\lambda}{4} \gg$ $D$, obtaining

$H(D, \lambda)=\frac{512 D^{2}}{15 \lambda^{3}}+O\left(\frac{D^{4}}{\lambda^{5}}\right)$,

hence

$\tilde{h}_{\mathrm{NN}(l w)}^{2}(\omega) \simeq \frac{32768 \pi G^{2} \Delta \tilde{M}^{2}(\omega)}{15 \omega^{4} \lambda^{6}} ;$

it seems at first surprising that the dependence on $D$ cancels out in the long wavelength approximation, whereas one could have expected to retain a dependence, which could lead to zero the noise in the $D \rightarrow 0$ limit case. However, we are actually in a situation in which the instrument is sensitive to the gradient of the gravity acceleration (see Eq. (23)), and therefore, barring other sources of noise, the sensitivity is independent on the baseline $D$.

We can now use Eq. (12) of [24] to relate the mass fluctuations with the measured seism

$\Delta \tilde{M}^{2}(\omega)=\frac{1}{16} \lambda^{6} \rho_{0}^{2}\left(\frac{\pi}{\lambda}\right)^{2} \tilde{x}_{\text {seism }}^{2}(\omega)$,

where $\rho_{0}$ is the density of the medium. We finally obtain

$\tilde{h}_{\mathrm{NN}(s w)}(\omega) \simeq \frac{2 \pi \sqrt{14 \pi} G \rho_{0}}{\sqrt{3} \omega^{2} D} \tilde{x}_{\mathrm{seism}}(\omega)$,

$\tilde{h}_{\mathrm{NN}(l w)}(\omega) \simeq \frac{16 \sqrt{2 \pi} G \rho_{0}}{\sqrt{15} \omega c_{L}} \tilde{x}_{\mathrm{seism}}(\omega)$,

where we have used the relation $\lambda \omega=2 \pi c_{L}$, with $c_{L}$ the speed of longitudinal seismic waves.

Comparing with Eq. (18) for the gravity gradient noise affecting the Virgo interferometer, we see that in the short wavelength limit, represented by Eq. (39), the frequency dependence (as expected) is the same. Instead, in the long wavelength limit Eq. (40), the $\mathrm{NN}$ affecting the atom interferometer has a slower growth for $\omega \rightarrow 0$, reflecting the presence of correlated noise at the two stations, that partially cancels out in Eq. (23).

We underline that this cancellation is not specific of a dual atomic interferometer: the same effect would occur in optical interferometers like Virgo, for shorter baselines. However, in optical interferometers long baselines are motivated by the need to reject the mirror position noise, which scales inversely with the distance: in atom interferometers some position noises, like the thermal noise, are instead expected to be absent, hence the baseline could be shorter.

In order to assess the significance of the cancellation effect, we choose favorable, yet realistic parameters: for the medium surrounding our hypothetical instrument, we assume a large $c_{L}=5000 \mathrm{~m} / \mathrm{s}$, characteristic of compact rock, and a density $\rho_{0} \simeq 2.7 \times 10^{3} \mathrm{~kg} \mathrm{~m}^{-3}$, a typical value for the continental crust; we also assume, on the basis of measurement taken in underground environments (for instance in the Kamioka mine [38] which will host KAGRA) a seismic noise $\tilde{x}_{\text {seism }} 10$ times lower than the one measured at the Virgo site (Eq. (20)).

We also assume to build a relatively large instrument, taking for the distance between the atom interferometers a value $D \simeq 1 \mathrm{~km}$ as proposed in [39]: we obtain

$\tilde{h}_{\mathrm{NN}(s w)}(\omega) \simeq \frac{10^{-18}}{[\omega /(2 \pi \mathrm{Hz})]^{4}} \mathrm{~Hz}^{-1 / 2}$,

$\frac{\omega}{2 \pi} \gg \frac{c_{L}}{D} \simeq 5 \mathrm{~Hz}$

$\tilde{h}_{\mathrm{NN}(l w)}(\omega) \simeq \frac{6 \times 10^{-19}}{[\omega /(2 \pi \mathrm{Hz})]^{3}} \mathrm{~Hz}^{-1 / 2}$,

$\frac{\omega}{2 \pi} \ll \frac{c_{L}}{4 D} \simeq 1.25 \mathrm{~Hz}$.

The resulting limit to the atom interferometer sensitivity is displayed in Fig. 4, over a frequency range which runs from the long to the short wavelength regimes; for comparison we display also the NN affecting the Virgo instrument; in the high frequency regime, the two curves differ just by a small scale, reflecting the different size of the instruments and the lower seismic noise anticipated for an underground atom interferometer. In the low frequency regime the residual correlation of the Newtonian noise which affects the two atom interferometers, thanks to the shorter baseline, contributes to a milder growth as $\omega \rightarrow 0$, and therefore leads to a sizable, though not dramatic, reduction of the noise over the Virgo case.

\section{Conclusions}

In this work we have evaluated the effect of fluctuations of the gravity field on the sensitivity of atom interferometers, 


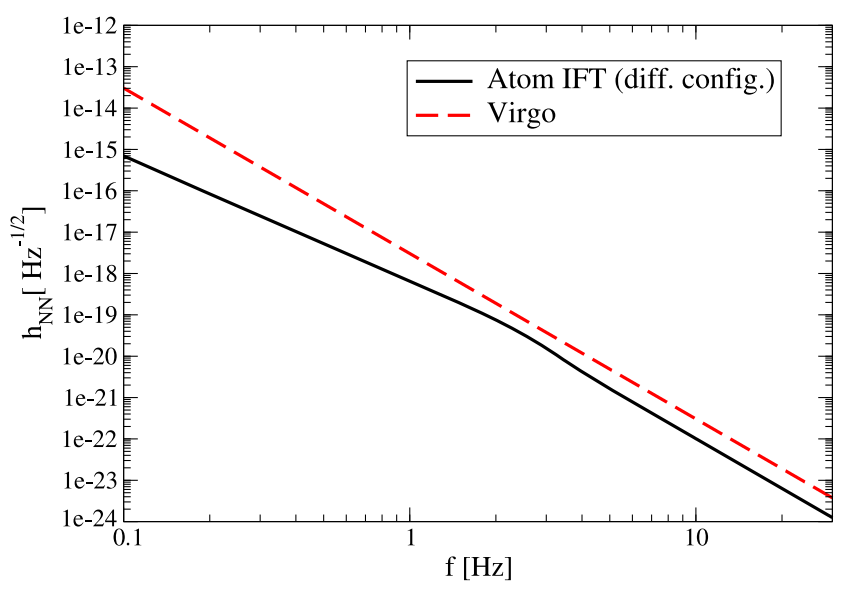

Fig. 4 Comparison of models of the Newtonian Noise as seen by the Virgo interferometer (dashed line) or by an hypothetical pair of atom interferometers operated in differential configuration (continuous line). Above a few Hz, the two curves run parallel, at different scales because of the different seismic noise (10 times lower for the hypothetical underground atom interferometers), and the different baseline of the two instruments ( $3 \mathrm{~km}$ for the length of Virgo arms, $1 \mathrm{~km}$ for the distance between the atom interferometers). At lower frequencies, thanks to its shorter baseline, the dual atom interferometer displays a different slope thanks to the cancellation effect

thus providing an estimate of the so-called Newtonian (or gravity gradient) noise for this kind of instruments.

We have seen that a mid-scale atom interferometer, with a baseline $L \sim 200 \mathrm{~m}$, is subject to a noise essentially equivalent to the one affecting a large scale optical interferometer, as Virgo.

We have also found that operating two small-scale atom interferometers, linked by a laser, at a larger distance $D \sim 1$ $\mathrm{km}$, in differential configuration, as proposed for instance in [39], there is an advantage at low frequency thanks to the residual Newtonian noise correlation and the resulting partial cancellation. However, the noise reduction is not dramatic and the Newtonian limit remains very significant: it is worth reminding that in order to detect a binary neutron star inspiral (say, at $z \sim 1$ ) sensitivities better than $10^{-22}$ would be required at $1 \mathrm{~Hz}$; even for larger systems, say a $1000 M_{\odot}$ binary black-hole coalescence, sensitivities of the order of $10^{-20}$ should be achieved, as discussed for instance in [40].

We conclude that, similarly to what is foreseen for future optical interferometers [11], operating successfully atom interferometers in the $[0.1,10] \mathrm{Hz}$ frequency window will require mitigating the gravity gradient noise; not just by choosing very quiet, underground sites, but also devising clever noise subtraction strategies.

We acknowledge that this study has a limitation in the model for the gravity fluctuations, which is approximate; however, as it has been the case for similar studies carried out for optical interferometers [23, 24], we believe that the use of more refined models will change the numerical re- sults only by small factors, which would not alter our conclusions.

Open Access This article is distributed under the terms of the Creative Commons Attribution License which permits any use, distribution, and reproduction in any medium, provided the original author(s) and the source are credited.

\section{References}

1. A. Accadia et al., (Virgo Collaboration) J. Instrum. 7, P03012 (2012)

2. http://www.geo600.org

3. B. Abbott et al., Rep. Prog. Phys. 72, 076901 (2009)

4. J. Abadie et al. (LIGO Scientific Collaboration Virgo Collaboration), Phys. Rev. D 85, 082002 (2012)

5. J. Abadie et al. (LIGO Scientific Collaboration Virgo Collaboration), Astrophys. J. 760, 12 (2012)

6. J. Abadie et al. (LIGO Scientific Collaboration Virgo Collaboration), Astrophys. J. 737, 93 (2011)

7. B.P. Abbott et al. (LIGO Scientific Collaboration Virgo Collaboration), Nature 460, 990 (2009)

8. G.M. Harry et al. (LIGO Scientific Collaboration), Class. Quantum Gravity 27, 084006 (2010)

9. https://wwwcascina.virgo.infn.it/advirgo

10. http://gwcenter.icrr.u-tokyo.ac.jp/en/

11. M. Punturo et al., Class. Quantum Gravity 27, 194002 (2010)

12. B. Sathyaprakash et al., Class. Quantum Gravity 29, 124013 (2012)

13. R. Spero, in Science Underground, Los Alamos, 1982. AIP Conf. Proceedings (AIP, New York, 1983)

14. P. Saulson, Phys. Rev. D 30, 732-736 (1983)

15. P. Berman (ed.), Atom Interferometry (Academic Press, New York, 1997)

16. F. Vetrano, in Aspen Winter Conference on $G W$ and Their Detection (2004). http://www.ligo.caltech.edu/LIGO_web/Aspen2004/ pdf/vetrano.pdf

17. R.Y. Chiao, A.D. Speliotopoulos, J. Mod. Opt. 51, 861 (2004)

18. A. Roura, D.R. Brill, B.L. Hu, C.W. Misner, W.D. Phillips, Phys. Rev. D 73, 084018 (2006)

19. P. Delva, M.C. Angonin, P. Tourrenc, Phys. Lett. A 357, 249 (2006)

20. G.M. Tino, F. Vetrano, Class. Quantum Gravity 24, 2167 (2007)

21. S. Dimopoulos, P.W. Graham, J.M. Hogan, M. Kasevich, S. Rajendran, Phys. Rev. D 78, 122002 (2008)

22. C.J. Bordé, Phys. Lett. A 140, 10-12 (1989)

23. S.A. Hughes, K.S. Thorne, Phys. Rev. D 58, 122002 (1998)

24. M. Beccaria et al., Class. Quantum Gravity 15, 3339-3362 (1998)

25. C.J. Bordé, Gen. Relativ. Gravit. 36, 475 (2004)

26. J.F. Riou, Y. Le Coq, F. Impens, W. Guerin, C.J. Bordé, A. Aspect, P. Bouyer, Phys. Rev. A 77, 033630 (2008)

27. C.J. Bordé, Eur. Phys. J. Spec. Top. 163, 315 (2008)

28. G.M. Tino, F. Vetrano, Gen. Relativ. Gravit. 43, 2037 (2011)

29. C.J. Bordé, Metrologia 39, 435-463 (2002)

30. F.K. Manasse, C.W. Misner, J. Math. Phys. 4, 735 (1963)

31. C.J. Bordé, C. R. Acad. Sci. Paris, t. 2, Ser. IV 509-530 (2001)

32. M. Punturo, The Virgo sensitivity curve. VIR-NOT-PER-1390-51. https://wwwcascina.virgo.infn.it/senscurve/VIR-NOT-PER-139051.pdf (1999)

33. T. Accadia et al. (Virgo Collaboration), J. Low Freq. Noise Vib. Act. Control 30, 63-79 (2011)

34. C.J. Bordé, J. Sharma, P. Tourrenc, T. Damour, J. Phys. Lett. 44, L983 (1983) 
35. H. Müller, S. Chiow, S. Hermann, S. Chu, Phys. Rev. Lett. 102, 240403 (2009)

36. M. Hoensee, S. Lan, R. Houtz, C. Chan, B. Estey, G. Kim, P. Kuan, H. Müller, Gen. Relativ. Gravit. 43, 1905 (2011)

37. J.M. Hogan, D.M.S. Johnson, S. Dickerson, T. Kovachy, A. Sugarbaker, S. Chiow, P.W. Graham, M. Kasevich, B. Saif, S. Rajendran, P. Bouyer, B.D. Seery, L. Feinberg, R. Keski-Kuha, Gen. Relativ. Gravit. 43, 1953 (2011)
38. M. Ohashi (LISM Collaboration), Laser interferometer in the Kamioka mine, in Proceedings of the 28th International Cosmic Ray Conference (2003). http://www-rccn.icrr.u-tokyo.ac.jp/ icrc2003/PROCEEDINGS/PDF/759.pdf

39. S. Dimopoulos, P.W. Graham, J.M. Hogan, M.A. Kasevich, S. Rajendran, Phys. Lett. B 678, 37 (2009)

40. S. Kawamura et al., J. Phys. Conf. Ser. 122, 012006 (2008) 